\title{
II. Referate aus geburtshülflich-gynäkologischen
}

\section{Gesellschaften}

I. Gesellschaft für Geburtshülfe und Gynäkologie zu Berlin.

8. V. (Ztschr. Bd. LXXVI. H. 3.) Demonstrationen. Bröse : Perforation des Uterus in der Schwangerschaft. Diskussion: Odebrecht, Bumm. - Runge: Zur Tiefenmessung von Röntgentiefenstrahlung. Diskussion: Bumm, Runge, Bröse. - Warnekros : a) Technische Neuerungen bei der Röntgentiefenbestrahlung; b) diirch Röntgenstrahlung geheiltes Cervixkarz $1 / 80 m$. Diskussion: Franz, Koblanck, Bröse, Haendly, Fromme, Jürsser, Hammerschlag, Bumm, Warnekros. -Franz : Mitteilung über Desinfektion der Haut. Diskussion: Fromme, Heymann, Franz, Jolly, Hammerschlag, Bumm, Franz. - Demonstration. Moraller : Cervixmyom. Diskussion: Franz, Moraller.

II. New York medizinische Akademie.

Sektion für Geburtshülfe und Gynäkologie. 24. XL (Med. Record. 16.1. S. 118.) Hellmann : Vorläufiger Berichfc über seine neue Methode für schmerzlose Geburten. Ch. Lieb: Physiologie und Pharmakologie des exstirpierten weiblichen Uterus. Diskussion: J. 0. Polak. 